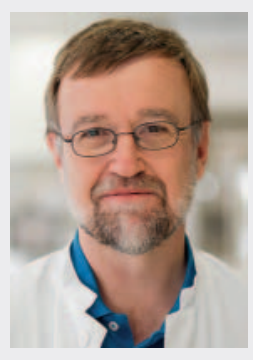

Prof. Dr. Hans-Joachim Mentzel

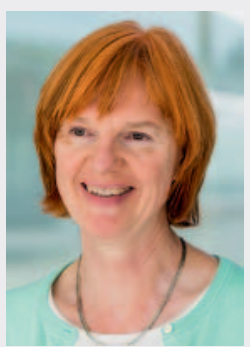

PD Dr. Thekla von Kalle

\section{Grußwort der Vorstandsvorsitzenden Prof. Dr. Hans-Joachim Mentzel und PD Dr. Thekla von Kalle zum Abstract-Band der GPR-Jahrestagung 2019}

\author{
Sehr geehrte Damen und Herren, \\ liebe Kolleginnen und Kollegen,
}

es ist uns als Vorstand der Gesellschaft für Pädiatrische Radiologie (GPR) eine große Freude, Ihnen diesen Abstract-Band zur wissenschaftlichen Entwicklung der Kinderradiologie als Publikation in der RöFo präsentieren zu dürfen.

Mit der hier vorgelegten, eigenständigen Abstract-Publikation zur 56. GPR-Jahrestagung werden wir 2 Zielen unserer Fachgesellschaft gerecht. Zum einen möchten wir (Nachwuchs-) Wissenschaftlern die Chance geben, sich auf prominente Weise mit ihren Forschungsarbeiten auf dem Gebiet der Kinderradiologie einem größeren Publikum vorzustellen.

Zum anderen geben wir über die Verbreitung dieses Abstract-Heftes an den großen Leserkreis der RöFo den Kolleginnen und Kollegen aller Teilgebiete der Radiologie einen Einblick in die Forschungs- und Weiterbildungsaktivitäten unseres Schwerpunktes. So enthält dieser Band zudem Kurzfassungen der Vorträge des Fortbildungstages und anderer eingeladener Vorträge. Vielleicht macht sich der ein oder andere von Ihnen nach anregender Lektüre der Abstracts auf den Weg zur Jahrestagung nach Rostock/ Warnemünde? Wir freuen uns ausdrücklich über Kongressteilnehmer aus dem Kreis der gesamten Radiologie!

Danke sagen möchten wir an dieser Stelle den eingeladenen Referentinnen und Referenten der Jahrestagung, die sich der zusätzlichen Mühe eines erweiterten Abstracts unterzogen haben. Wir wissen diesen zusätzlichen Aufwand sehr zu schätzen. Dank gilt allen Kolleginnen und Kollegen, die in den Schwerpunkten der Jahrestagung „Pädiatrische Neuroradiologie“, „Interventionen“, „Muskuloskelettale Bildgebung“ sowie „Strahlen- und Kinderschutz“ wissenschaftliche Arbeiten eingereicht haben bzw. Kasuistiken vorstellen werden.

Ein großes Dankeschön gilt schließlich dem Thieme-Verlag Stuttgart für die umfangreiche und zuverlässige Betreuung dieses „Erstlings“.

Im Namen des gesamten Vorstands und der Kongresspräsidentin Dr. Christina Hauenstein, die ein hervorragendes Programm vorgelegt hat, freuen wir uns, Sie vom 18.-21.09.2019 in Rostock/Warnemünde begrüßen zu dürfen.

Mit kollegialen Grüßen

Prof. Dr. Hans-Joachim Mentzel

1. Vorsitzender

PD Dr. Thekla von Kalle

2. Vorsitzende 